\title{
Editorial Keine Arbeit - kein Problem?
}

Angetrieben von hohen Exportüberschüssen geht der ökonomische Aufschwung in der Bundesrepublik in sein achtes Jahr. Auch wenn sich die Zuwachsraten des Sozialproduktes im Zyklendurchschnitt auf einem historisch relativ niedrigen Niveau bewegen, handelt es sich doch um den zeitlich längsten Aufschwung der Nachkriegsgeschichte. Eine ideale Konstellation dafür, so sollte man meinen, daß sich der insbesondere von Konservativen beschworene Zusammenhang von Wachstum der Produktion und der Beschäftigung auch in einen Rückgang der Arbeitslosenzahlen übersetzt? Tatsächlich aber ist die Zahl der offiziell registrierten Arbeitslosen nur geringfügig zurückgegangen. Die allmonatlichen Fernsehauftritte des Präsidenten der Nürnberger Bundesanstalt, Franke, verheißen zwar periodisch Licht am Ende des Tunnels - allein es handelt sich dabei offensichtlich im Irrlichter, die als Hoffnungsschimmer zu erkennen es einer besonderen Begabung bedarf. Anders als noch zu Beginn der siebziger Jahre, als das Überschreiten der Millionen-Grenze Nahrung für vielfältige Spekulationen über die Grenzen politischer Legitimität und sozialer Stabilität der westdeutschen Gesellschaft bot, hat sich die Öffentlichkeit hierzulande offensichtlich mit der Existenz von Massenarbeitslosigkeit auf hohem Niveau arrangiert. Daran haben weder gewerkschaftliche Versuche, den Dauerskandal Massenarbeitslosigkeit zu thematisieren, noch die politischen Selbstorganisationsversuche von Arbeitslosen etwas ändern können.

Deutlich wurde dieser Einstellungswandel der Öffentlichkeit an zwei Ereignissen der jüngsten Zeit: Zum einen an der von der Bundesanstalt für Arbeit fast klammheimlich vorgenommenen Veränderung der Berechnungsgrundlagen der Arbeitslosenstatistik; durch statistische Manipulation wurde so die Zahl der Arbeitslosen geschönt, ohne daß dieser Etikettenschwindel zu größeren Diskussionen über oder gar öffentlichen Protesten gegen die damit beabsichtigte Legitimation der sozialen Schließung des Beschäftigungssystems geführt hätte. Ein zweites Beispiel bietet die jüngste Fluchtwelle von DDR-Bürgern, die nahezu unisono als leistungsfähige, einsatz- und mobilitätswillige, disziplinierte Arbcitskräfte willkommen geheißen werden. Auch wenn dabei deutsch-deutsche Besonderheiten eine Rolle spielen mögen, darf es doch erstaunen, wie die überwiegend jungen Menschen als dringlichst benötigte Arbeitskräfte angesichts des westdeutschen Reservoirs an Anbietern von Arbeitskraft in Empfang genommen werden. Die Bilder von den »Auffanglagern«, in den ökonomisch weniger entwickelten Randzonen der BRD gelegen, wo klein- und mittelständische Unternehmer höchstpersönlich Arbeitskräfte werben und neben Arbeitsplätzen auch gleich Wohnungen anbieten, sollten angesichts von 1,8 Millionen registrierten Arbeitslosen doch zumindest Überraschung auslösen. Dies um so mehr, als mit den auf relativ niedrigem technologischen Niveau qualifizierten Arbeitskräften - so jedenfalls die weit- 
geteilte Einschätzung der DDR-Industrie durch westdeutsche Politiker und Industrielle - die allenthalben beschworene Facharbeiterlücke schwerlich geschlossen werden dürfte.

In den Meinungsumfragen wird das Problem der Arbeitslosigkeit auch Ende der achtziger Jahre zwar weiterhin als eines der drängendsten gesellschaftlichen Probleme bezeichnet. Das dürfte aber eher Ausdruck einer Art schlechten Gewissens sein denn wirkliche Einsicht in die unfaire und inhumane Verteilung von Arbeit und daher von Lebenschancen in einer Gesellschaft, in der die Teilhabe am Beschäftigungssystem über Einkommen, Prestige und individuelle Zukunft entscheidet. Praktisch-politisch hat diese Prioritätensetzung jedenfalls wenig Konsequenzen - selbst wenn das Stabilitäts- und Wachstumsgesetz von 1966 dem Wortlaut nach Vollbeschäftigungspolitik zu einer vorrangigen Aufgabe der Bundesregierung erklärt. Tatsächlich handelt es sich in diesem Gesetz mit dem lange gefeierten $\gg$ modernsten wirtschaftspolitischen Instrumentarium der Welt « (Herbert Ehrenberg) um eine äußerst weiche Verpflichtung, die außerdem noch durch die historische Bindung an andere makroökonomische Konstellationen (Preisentwicklung; Zahlungsbilanz etc.) eingeschränkt wird. Die theoretisch begründete Vorstellung, daß die Beschäftigungsmenge von der effektiven Nachfrage bestimmt sei, das Preisniveau von der Lohnhöhe und die Leistungsbilanz vom Wechselkurs, und die Wirtschaftspolitik sich daher eben dieser Variablen bedienen könne, mußte angesichts veränderter globaler Wachstumsbedingungen und neuer Akkumulationsstrategien der Unternehmen (Industriekonzerne wie Banken) wohl oder übel ad acta gelegt werden. Schmerzlich erfahren hat das bereits die SchmidtRegierung, deren wirtschaftspolitisches Menu aus Nachfrage- und Angebotspolitik bis zuletzt - freilich halbherzig - auf den mittel- oder langfristig wirkenden keynesianischen Mechanismus setzte. Realistischer (und brutaler für die Arbeitslosen) handelte die liberal-konservative Koalition aus CDU/CSU/FDP, die zwar der Arbeitslosigkeit rhetorisch den Kampf ansagte, aber gleichzeitig darauf bestand, den Staat von seiner politischen Vollbeschäftigungsverantwortung zu entbinden. Mit der »Privatisierung des Beschäftigungsrisikos« gelang es der Regierung auch, sich den »Legitimationszwängen zu entwinden « (Habermas). Über Beschäftigung werde am Arbeitsmarkt entschieden, so die anti-keynesianische Botschaft, und dort herrsche die Tarifautonomie. Daß die Käufer und erst recht die Verkäufer von Arbeitskraft nicht die Mittel und Instrumente kontrollieren, mit denen Vollbeschäftigung potentiell verwirklicht werden könnte, wurde dabei - bewußt - ausgeblendet.

Heute läßt sich festhalten, daß diese Strategie der Privatisierung des Beschäftigungsrisikos weitgehend erfolgreich war. Indem die Entscheidung über Arbeitsplatz und Arbeitslosigkeit ideologisch wie praktisch wieder dem Arbeitsmarkt und damit den Tarifparteien, also den Organisationen von Kapital und Arbeit, sowie dem individuellen Anbieterverhalten zugewiesen wurde, erfolgte nicht allein eine Entlastung des politischen Systems, sondern es wurden auch Diskurse gesellschaftlich aufgewertet, die zuvor allenfalls der Stammtischseligkeit zugestanden waren. Die Opfer des kapitalistischen Arbeitsmarktes wurden zu Tätern umdefiniert, die sich - womöglich sogar aus rationalen Kalkülen - in der »sozialen Hängematte« ausruhen und sich ihre 
Versicherungsleistungen in südlichere Gefilde überweisen lassen (vgl. dazu Moser/ Zilian). Wissenschaftlichen Anstrich erhalten diese Diskurse von seiten so mancher Perle der ökonomischen Profession, wenn diese das Problem der Massenarbeitslosigkeit mit zu vielen »Marktrigiditäten « zu klären vorgibt: Weil das freie Schalten und Walten des Arbeitsmarktes durch politische Regulierungen beeinträchtigt sei, gelte es zu deregulieren, um Leistung und Wettbewerb in ihr Recht zu setzen. Vollbeschäftigung sei in einer Gesellschaft mit »grundsätzlich stabilem privaten Sektor « zu schaffen, wenn Löhne und Leistung wieder zusammenpassen: durch generelle Senkung der Reallöhne und insbesondere durch stärkere Differenzierung der Löhne entsprechend Qualifikation und Leistung. Arbeitslosigkeit als gesellschaftliches Problem existiert demnach nicht, und eine politische Aufgabe läßt sich mit einem Nicht-Problem schlecht begründen, da nichts durch den Staat zu lösen ist.

Dies war nicht immer so. Als zu Beginn der 50er Jahre mehrere Millionen Arbeitslose gezählt wurden, mußte sich die Politik trotz der neoliberalen Rauchwolken über den offiziellen Konzepten - man lese die Sonntagsreden des »Vaters des Wirtschaftswunders« Ludwig Erhard nach - höchst aktiv auf die Schaffung von Arbeitsplätzen konzentrieren. Doch damals war das weltwirtschaftliche Umfeld günstig, die Schranken der Akkumulation von Kapital waren außerhalb des Horizonts, konjunkturelle Krisen oder gar strukturelle Krisen schienen abgeschafft. Jede staatliche Förderung von privaten Investitionen resultierte in neuen Arbeitsplätzen; trotz Flüchtlingszustrom wurde die Vollbeschäftigung schnell erreicht. Mit den veränderten Bedingungen ökonomischen Wachstums, insbesondere mit der vielfach analysierten »Entkoppelung von Wachstum und Beschäftigung « jedoch können die damals wirksamen Therapien nicht mehr angewandt werden. Anstatt sich der theoretischen und politischen Anstrengung zu unterziehen, Konzepte einer Vollbeschäftigungspolitik unter den veränderten ökonomisch-sozialen Bedingungen der späten 80er Jahre zu entwickeln, hält sich der "main stream « der ökonomischen Theorie an die gesellschaftliche Umdeutung des Skandals Massenarbeitslosigkeit in gesellschaftliche Normalität: Weil Massenarbeitslosigkeit nicht länger ein gesellschaftliches Problem sei, brauche die ökonomische Theorie sich auch nicht weiter darum zu kümmern. Soweit war man - wie Zsuzsa Ferge zeigt - unter ganz anderen politisch-ideologischen Vorzeichen in Ungarn auch schon einmal. Dennoch ist Arbeitslosigkeit als offenes gesellschaftliches Problem auf die Tagesordnung zurïckgekehrt - ohne daß sich die Mittel zu seiner Bearbeitung verbessert hätten.

Die gesellschaftliche Dethematisierung von Arbeitslosigkeit ist freilich keineswegs allein das Produkt ideologischer Konflikte und deren sozialer Verdrängung. In wesentlich größerem Maße dazu beigetragen haben dürfte in der BRD die besondere Strukturierung von Arbeitslosigkeit. Wie Hinrichs zeigt, verweisen die hohen $\mathrm{Zu}$ - und Abgangszahlen im Bestand der registrierten Arbeitslosen zum einen auf eine ausgeprägte Durchlässigkeit und Flexibilität der Arbeitsmärkte; zum anderen drückt sich darin aber auch eine hohe kumulative und Mehrfacharbeitslosigkeit aus: Das Risiko des Eintritts in die Arbeitslosigkeit ist seit Mitte der siebziger Jahre steil angewachsen, die eigentliche »Last« der Arbeitslosigkeit wird aber von einer relativ kleinen 
Gruppe der Lohnabhängigen getragen. Diese spezifische Risiko- und Lastenverteilung dürfte die praktizierten Dethematisierungsstrategien ebenso erleichtert haben wie die sektoral und regional gespaltene Akkumulationsentwicklung und der Trend zur »neuen Selbständigkeit« (vgl. Bögenhold), also zu einer »Informalisierung« der Arbeit, wie sie in allen kapitalistischen Gesellschaften mehr oder weniger ausgeprägt ist. So erklärt sich auch die Gleichzeitigkeit von Freisetzung und Knappheit von Arbeitskräften, das verwirrende Bild von Überschuß und Mangel am Arbeitsmarkt. Dadurch wird Interpretationen Vorschub geleistet, denenzufolge angesichts des Bedarfs an Arbeitskräften jeder, der arbeiten wolle, auch einen Arbeitsplatz erhalten könne wie bei der Diskussion um die Beschäftigung der DDR-Flüchtlinge betont wird. Also: Die registrierten Arbeitslosen seien mithin »Schmarotzer «, die mit Zahlungsentzug oder wenigstens Zahlungskürzungen bestraft werden sollten.

Arbeitslosigkeit ist nicht allein das Problem der Arbeitslosen oder der Lohnabhängigen, die hohe Arbeitsmarktrisiken aufweisen, sondern, wie Ganßmann zeigt, auch ein Problemfür die heute beschäftigten Lohnabhängigen: Mit der Massenarbeitslosigkeit gehen Sog-Effekte einher, die die Verhandlungsposition von Gewerkschaften schwächen und die soziale Lage auch relativ privilegierter Gruppen von Lohnabhängigen negativ berühren. Es liegt auf der Hand, daß solche Sog-Effekte den Nährboden für solidarisches Handeln aller Lohnabhängigen bilden (könnten). Insbesondere die Strategie der Wochenarbeitszeitverkürzung auf 35 Stunden hat sich diesen Zusammenhang zu eigen gemacht und ausdrücklich den trade-off von Arbeitszeitverkürzung und (Nominal-) Lohnsteigerung in Kauf genommen, um die Schaffung neuer Arbeitsplätze zu erzwingen. Trotz aller Quantifizierungs- und Zurechnungsprobleme dïrfte der Beschäftigungseffekt dieser Strategie unbestreitbar sein. Auf der Hand liegt aber auch, daß einer solchen Solidaraktion enge Grenzen gesteckt sind, wenn sie nicht von einer gleichgerichteten staatlichen Politik flankiert wird, die das Interesse der $\gg \mathrm{Ar}-$ beitgeber « an einem - wie auch immer definierten - Mindestsockel an Arbeitslosigkeit ebenso einschränkt wie sie die Ausweitung prekärer Beschäftigungsverhältnisse verhindert.

Die Existenz von Massenarbeitslosigkeit ist kein Indiz für eine Krise des Kapitalismus sondern zunächst einmal ein Ausweis für die Entwicklung der Produktivkräfte. Dies gilt jedenfalls dann, wenn im Zuge durchschnittlicher Auslastung der Produktionskapazitäten Arbeitskräfte aus dem Produktionsprozeß »ausgeschwitzt« werden, weil die Produktivität der Arbeit zugenommen hat. Auch gilt es spätestens seit Marx in Rechnung zu stellen, daß die Kapitalisten als Klasse durchaus Interesse an einer Mindestgröße der industriellen Reservearmee - natïrlich nach Qualifikationsmerkmalen differenziert - haben, weil die Konkurrenz zwischen beschäftigten und arbeitslosen Lohnabhängigen zu einem Druck auf die Löhne und zu erhöhter Arbeitsdisziplin und Arbeitsleistung führt. Wenn man die Funktionslogik kapitalistischer Gesellschaften in Rechnung stellt, ist mithin Arbeitslosigkeit der Normalfall und Vollbeschäftigung die historische Ausnahme. Innerhalb dieser Rahmenbedingungen politisch Vollbeschäftigung einzufordern, heißt deshalb nichts weniger, als den Ausnahme- zum Normalfall zu machen. Daß dies kein aussichtsloses Unterfan- 
gen sein muß, belegen die - in ihrer Ausgestaltung gegensätzlichen - Fälle Schweden und USA. In der politischen Landschaft der BRD haben beide Fälle ihre Vertreter: Während die liberal-konservative Regierung in ihrer Mehrheit dem US-Vorbild deregulierter und hochmobiler Arbeitsmärkte nachhängt und von der Medizin Reagans auch in der BRD Linderung erhofft, träumt die Mehrheitssozialdemokratie bis heute von einer politischen Vollbeschäftigungsgarantie, die durch ein hohes Maß an Regulierungen - wie in Schweden - gewährleistet werden solle. Beim schwedischen Modell ergibt sich allerdings das Problem, inwieweit es verallgemeinerbar oder zumindest auf die Bundesrepublik übertragbar ist. Aber auch davon abgesehen muß die Frage aufgeworfen werden, ob Vollbeschäftigung noch in erster Linie quantitativ oder nicht auch qualitativ definiert werden muß. Vor bereits mehr als zehn Jahren hat ja Joan Robinson von der »zweiten Krise der Nationalökonomie « gesprochen: es gelte heute nicht mehr wie noch zu Keynes Zeiten, überhaupt Arbeitsplätze anzubieten, sondern solche mit entsprechender Qualität (Arbeitsbedingungen, ökologische Verträglichkeit etc.). Auch ist in Rechnung zu stellen, daß Vollbeschäftigung mit mies bezahlten, sozial nicht abgesicherten Jobs (Reagans »Beschäftigungswunder « in den USA) etwas ganz anderes ist als Vollbeschäftigung unter Wahrung des Normalarbeitsverhältnisses. Die von Hinrichs angedeutete und in der letzten Ausgabe der PROKLA von Mückenberger breiter diskutierte Strategie einer Entkoppelung von Beschäftigung und Einkommen verweist auf einen dem Niveau der Reichtumsproduktion der Bundesrepublik angemessenen Weg, Lebens- und Entwicklungschancen von Individuen zu verbessern. Aber auch für eine solche Strategie gilt, daß sich erst gesellschaftliche Koalitionen herausbilden müssen, die in der Lage sind, die entstehenden Kosten der Vollbeschäftigung neu zu verteilen, bevor deren individuelle und soziale Gratifikationen eingestrichen werden können. 